EOMmun Communication et organisation

Or

Recherches émergentes en communication des organisations

\title{
L'intelligence du social dans les organisations
}

The intelligence of social issues in organizations

Jacques Bonnet

\section{CpenEdition}

Journals

Édition électronique

URL : http://journals.openedition.org/communicationorganisation/4897

DOI : 10.4000/communicationorganisation.4897

ISSN : 1775-3546

Éditeur

Presses universitaires de Bordeaux

Édition imprimée

Date de publication : 1 juin 2015

Pagination : 63-74

ISSN : $1168-5549$

\section{Référence électronique}

Jacques Bonnet, "L'intelligence du social dans les organisations », Communication et organisation [En ligne], 47 | 2015, mis en ligne le 01 juin 2018, consulté le 02 mai 2019. URL : http://

journals.openedition.org/communicationorganisation/4897 ; DOI : 10.4000/

communicationorganisation.4897 


\title{
L'intelligence du social dans les organisations
}

\author{
Jacques Bonnet ${ }^{1}$
}

Je voudrais tout d'abord préciser les orientations de recherche de l'équipe de recherche Cimeos/Cosmos que je dirige à l'Université de Bourgogne et qui a pu bénéficier, depuis de nombreuses années, du riche et fécond support d'expression scientifique constitué par la revue Communication E'Organisation. Pour l'équipe Cosmos, l'analyse et la modélisation des processus et des formes communicationnels appellent la mise en dialogue de ces trois angles de lecture que sont les organisations, les territoires et les savoirs. L'équipe propose ainsi une recherche incarnée et contextualisée susceptible d'éclairer les changements sociotechniques, socio-économiques et culturels en cours.

\section{Trois grandes thématiques de recherche}

Les organisations (entreprises, administrations, collectivités territoriales, associations, réseaux,...) et leur communication sont appréhendées d'un point de vue contextuel et processuel. Les mutations organisationnelles sont étudiées non d'un point de vue structurel ou gestionnaire, mais en tant qu'espaces d'interactions où, et à partir desquels, s'élaborent, se nouent et s'expriment des perceptions, des représentations individuelles et collectives, des systèmes de valeurs, des attributions, des constructions identitaires ainsi que des dispositifs communicationnels, au regard d'activités (production, services, éducation, santé,...) et de changements sociotechniques et culturels. Nous considérons les organisations comme des espaces de conception, de diffusion et d'échanges mais aussi de confrontation de significations individuelles et collectives. De ce point de vue, les organisations posent, selon nous, la question du rapport dialogique entre prescription, activité et logiques d'acteurs, rapport qui pose la problématique plus globale du sens que les individus et les groupes trouvent et attribuent à leurs activités et à leur inscription dans les espaces sociaux et temporels où s'inscrivent leurs activités.

Les territoires sont considérés, au-delà d'une conception spatiale, au plan de leur définition représentationnelle, des usages et des dispositifs qui leur sont

1 Professeur émérite en sciences de l'information. et de la communication, Agrosup Dijon/Cimeos (EA 4177) Université de Bourgogne. 
liés, des formes d'identité et d'appartenance qui les sous-tendent, des valeurs qu'ils expriment, des mutations communicationnelles qu'ils expérimentent, notamment en termes de groupes d'entraide, de réseaux, de communautés sociales, professionnelles ou culturelles, formelles ou virtuelles. Il s'agit donc tout particulièrement, à partir des concepts d'ancrage, de développement et d'intelligence territoriale, d'identifier les évolutions propres aux conceptions, aux formes et aux usages du territoire, donc les mouvements et les dynamiques de sens autour de ce référent essentiel de la communication humaine qu'est le concept de territoire.

La notion de savoir(s) est abordée selon une approche qui ne s'inscrit pas dans un ancrage déclaratif ou académique mais qui s'intéresse aux processus d'élaboration et de partage de significations au regard d'une réalité porteuse de connaissance(s) et exigeante en habiletés. Le savoir et les savoirs - professionnels, populaires, artisans, scientifiques, technologiques ou autres - sont approchés en tant que processus et formes d'échange et de confrontation de représentations entre les acteurs, en tant que processus et formes de médiation face à des situations, des événements et des informations marqués par l'incertitude et la complexité, dans un environnement de travail, de formation, de soin, d'entraide, dans la sphère publique et la sphère privée.

Jeux et enjeux de savoirs dans les communications et les organisations : quelques précisions sur les recherches menées au sein de Cosmos.

Le savoir se trouve aujourd'hui questionné, interpellé, car il est objet de doute, voire de suspicion au sein de sociétés confrontées à des incertitudes socio-économiques de toutes natures et à la complexification des organisations, des activités et des relations sociales. Face à cette montée des incertitudes qui fait suite aux Trente Glorieuses, trente années d'expansion socio-économique post-seconde guerre mondiale, le savoir semble en effet moins lié à l'idéal de progrès porté ou sous-entendu jadis par la modernité industrielle ${ }^{2}$, ce qui conduit nombre d'auteurs, à l'instar de Gilles Lipovetsky, à parler d'une société déceptive (Lipovetsky 2006), c'est-à-dire et entre autres, d'une société en proie au doute et à la suspicion quant à la plus-value potentielle pouvant résulter de l'acquisition de savoirs. La complexification évoquée ici se caractérise notamment par le développement exponentiel des interactions entre des acteurs individuels ou collectifs de plus en plus en mesure de connecter leurs expériences, leurs désirs, leurs pratiques, leurs cultures, leurs logiques et de réagir en direct aux évènements du monde tout en mettant en scène leurs connaissances et même leur propre vie. Les sociétés occidentales se trouvent également marquées par la multiplicité et la différenciation des sources et des vecteurs de savoir(s).

2 période correspondant, dans la plupart des publications qui l'évoquent, à plus de deux siècles depuis la Révolution industrielle née en Grande-Bretagne et la Révolution française de la fin du 18ème siècle jusqu'aux Trente Glorieuses, années d'expansion économique (1945-1975). 
Parmi les questions que nous posons figurent celles-ci : Peut-on ainsi vraiment parler de nouveaux modèles de transmission et de partage du savoir et en quoi ? Si l'on retient cette hypothèse, par quels processus ces derniers prennent-ils forme et sens, comment s'ancrent-ils ou traversentils les usages, les pratiques, les cultures ? Ces savoirs préfigurent-ils ou engagent-ils des maillages jusqu'alors restreints et contraints par la logique structurante des disciplines d'enseignement ou des supports de diffusion ? Par exemple, les savoirs portés par le courant du développement durable rencontrent-ils les principes posés par la gouvernance et l'e-gouvernance ? Plus globalement, en quoi, pourquoi et comment les organisations publiques et privées (entreprises, administrations, collectivités territoriales, associations et organismes) intègrent-elles ou non cette dimension du et des savoirs dans leur politique, leurs choix stratégiques, leurs activités, leur image et leurs messages en général ? Sur le plan interne des organisations, la capitalisation et le croisement des expériences ont-ils une autre signification que la simple ambition managériale d'obtenir une performance collective ?

Voilà pour une rapide présentation des principales questions de recherche qui nous occupent.

\section{Pourquoi faire de l'intelligence $d u$ social un axe de recherche ?}

Pour en venir au thème central de cette intervention, de quoi tentonsnous de parler et qu'étudions-nous lorsque nous abordons cette question de l'intelligence $d u$ social dans les organisations et non de l'intelligence sociale, notion qui ferait courir le risque d'une approche finie incompatible à nos yeux avec le contexte d'imprévisibilité et de complexité dans lequel s'inscrivent la plupart des discours et des pratiques que nous étudions.

Tout d'abord, pourquoi ne parlerait-on pas d'intelligence $d u$ social alors que l'on traite avec tant d'insistance d'intelligence économique, d'intelligence numérique, d'intelligence territoriale et même d'intelligence artificielle ?

Bien sûr le terme "intelligence " pose déjà problème en lui-même.

Je n'ai guère le temps de faire ici une revue des travaux concernant ou approchant cette délicate notion d'intelligence $d u$ social qui est au centre de mes recherches et que je tente de développer et de nourrir, mais afin de l'éclairer, je ferai référence à un ouvrage de la fin des années 1990, dans lequel J. M. Berthelot (Berthelot 1997) posait dans le cadre d'une approche sociologique de l'éducation le problème de l'intelligence $d u$ social. Il installait notamment le concept d'intelligence à travers cette définition : " la capacité d'établir des liens et de saisir des relations ». Des liens, des relations : une approche quasi-communicationnelle dans sa formulation traduite ensuite majoritairement en repérage de schèmes, de logiques de lectures, comme matrices d'explication articulant différents processus :

1-de dépendance (causal, fonctionnel),

2-de signification (jeu des représentations et des codes sémiotiques), 
3-et enfin de composition en termes de changement et d'histoire en train de se faire.

Les liens entre ces logiques de dépendance, de signification et de composition ne peuvent nous échapper, ce qui nous conduit à poser d'un point de vue sociocognitif, la question de l'intelligence $d u$ social hors de toute tentative déterministe et prescriptive, mais plutôt comme une activité de connaissance en mouvement et sujette à une contextualisation et à une recomposition permanentes.

Pour nous, parler d'intelligence $d u$ social, consiste à s'intéresser à ces postures, ces habiletés, ces modes et ces logiques d'intervention individuelles et collectives qui nourrissent, facilitent et rendent fécondes les interactions sociales dans les contextes que nous étudions : que ce soit en milieu de santé, de production de biens ou de services, de formation ou autre. Interaction, médiation, négociation, coopération, coordination, altérité, intercompréhension, voici des questions neuves ou renouvelées dans des contextes sociétaux et organisationnels qui ont du mal à s'en saisir, notamment du fait de leur ancrage fonctionnaliste et de leur difficulté à entrer dans l'ère de la complexité et de l'incertitude. C'est sur ces questions que peuvent se refonder les principes d'appartenance, d'identité, de groupe, de communauté, de réseau, et pour tout dire de Cité, bref toutes ces questions qui tendent à construire une dialogique de la relation et à rendre lisibles et peut-être possibles des capabilités individuelles et collectives. Je reprends ici, comme je l'ai fait à Dijon lors d'un colloque intitulé "Acteurs, auteurs, spectateurs " (Bonnet, Bonnet, Raichvarg 2014), ce néologisme proposé par Amartya Sen, auteur de "L'idée de Justice » (Sen 2010). Capabilité est un terme qui associe la notion de capacité et celle de liberté d'agir et d'intervenir. Telle est la question : quelle place et quels rôles les organisations accordent-elles à ces capabilités individuelles et collectives combinant capacité et liberté d'agir, au regard de quelles conceptions et de quelles finalités? Il s'agit donc d'aborder le social comme un construit fondé sur cet ensemble d'interactions et sur ces capabilités invitées ou non à s'exprimer dans l'agir et le vivre ensemble.

Quelles sont les questions que peut poser la notion d'intelligence $d u$ social en matière de recherche en Sciences de l'information et de la communication dans le contexte des organisations, au cœur des pratiques et des discours relevés et observés et en quoi tout cela a-t-il constitué et constitue-t-il une contribution à la revue que nous célébrons ici ? Je me limiterai à relever quelques-unes de ces questions, en tentant de les relier à quelques «éléments observables ».

\section{Des questionnements multiples}

Notons tout d'abord que la notion d'Intelligence $d u$ social questionne la capacité des modèles organisationnels d'essence principalement gestionnaire et transmissive à accueillir la question du social, à prendre en compte le champ 
du social. Il s'agit en quelque sorte et pour reprendre l'expression de Zygmunt Bauman (Bauman 2007), de questionner la modernité organisationnelle " liquide ». Lintelligence $d u$ social propose un changement de paradigme pour envisager la capacité des organisations à se transformer sous l'influence des contraintes et des enjeux externes : Bauman nous dit que les déformations que connaissent les liquides sont dues à des liens entre leurs particules qui sont trop faibles pour résister aux diverses pressions. En conséquence, pour nous, les organisations ont-elles une plasticité voulue ou subie face aux pressions du social ? Si oui, cette plasticité peut-elle être analysée et modélisée ? Ainsi, les organisations peuvent-elles conserver leurs formes sous la pression de forces externes, qu'est-ce qui peut permettre ou empêcher cette conservation ou favoriser ces transformations ? La question peut tout autant être rapportée à des organisations qui vivent des pressions internes, par exemple dans le cadre de nouvelles orientations de structures, de métiers, de valeurs. Plus globalement, l'héritage organisationnel des modèles issus de la modernité industrielle rend-il possible cette prise en compte du social vers ce que l'on a pu parfois dénommer des organisations communicantes?

Par ailleurs, le concept d'intelligence $d u$ social conduit à considérer la dynamique complexe et mouvante des rapports entre le Réel, le Symbolique et le Sensible dans les organisations, rapports eux-mêmes sous l'influence de contextes socioprofessionnels et culturels en mutation.

Il s'agit donc de prendre en compte, au sein des organisations, la distinction entre le réel d'une part, et, d'autre part, le sensible (perception, représentations, valeurs, attributions, identités) et le symbolique (signes et formes communicationnels). Il s'agit non pas de confondre mais de mettre en système ces trois fondements de l'agir individuel et collectif et surtout de considérer leurs interactions dans des contextes eux-mêmes influents. Illustrons cela par un constat issu de nos recherches : nombre de décisions sont prises aujourd'hui dans les organisations à l'aune d'une rationalisation poussée qui pense échapper à l'incertitude et à la complexité induites par les événements socio-économiques et par la perception mouvante des acteurs, en tentant de les réifier, en tentant de les " contrôler " comme si la dimension du social était par nature contre-productive ou constituait une perte de temps et d'efficacité. Des entretiens semi-directifs menés auprès de personnels lors de changements structurels ainsi que les analyses menées sur des corpus de projets d'entreprises, d'organismes, de collectivités, montrent cette étrangeté qu'est parfois l'absence des acteurs lorsqu'il s'agit de changements qui pourtant les concernent et les impliquent. Ici, c'est la place réservée aux acteurs dans la conception politique des changements qui mérite étude et analyse dans sa nature et ses effets. L'analyse des fameuses résistances aux changements vient d'ailleurs souvent corroborer ce constat et on remarquera que cette « résistance au changement " semble posée et évaluée le plus souvent du point de vue de la seule prescription et sur la base d'une hypothèse qui pose la résistance 
en question comme inéluctable. Comme le note Alain Ehrenberg, « ce n'est pas parce qu'aujourd'hui les choses semblent plus personnelles qu'elles sont moins sociales. On a affaire à un changement de l'esprit des institutions, qui est justement ce changement dans nos manières d'agir » (Ehrenberg 2010).

Et d'ailleurs où est la dimension politique de la Cité-organisation qui pourtant aime tant parler de stratégie, de communauté de valeurs ou de destin ou encore de projet engageant l'ensemble de ses acteurs ? Du spectateur à l'acteur voire à l'auteur, ce sont des soubassements conceptuels et/ou idéologiques qui posent l'hypothèse d'une passivité, d'une participation ou bien d'une réelle implication des acteurs. D'où la problématique générale de la participation trop souvent ramenée à des questions d'intéressement aux résultats, à des formes d'animation sociale plus ou moins appréciées des personnels qui désertent les cérémonies de vœux,... et à une kyrielle d'outils et de supports sensés créer une nouvelle efficience collective à l'aune d'un sentiment d'appartenance reboosté par la communication interne, d'où le regain d'intérêt actuel dans les entreprises pour ces questions à grand renfort d'outils en tous genres.

Afin de poser les questions d'élaboration du sens de l'action humaine en tant que rapport au réel, il s'agira de proposer une traduction méthodologique à travers les notions de prescription, d'activité et de représentation d'acteurs. Par prescription, nous entendons les commandes plus ou moins formelles d'activité, d'intervention ou de comportement, commandes dont l'origine peut être hiérarchique du fait de décideurs, ou bien réglementaire ou encore se situer dans le registre d'un changement de politique ou de technicité ou de tout autre événement appelant une réponse en termes de production de sens par des acteurs invités ou contraints à agir. Répondre à des événements, au sens phénoménologique du terme, constitue à nos yeux un des enjeux essentiels actuels pour toute organisation et ses acteurs dans le contexte de complexité et d'incertitudes croissantes qui marquent les activités humaines.

Par ailleurs, nous entendrons par activité l'observable des usages, des pratiques dans le champ du travail, de la formation, de la consommation d'information, de services ou de produits.

Quant à la question des représentations, nous la resituerons au regard de la définition posée par Abric : « une activité mentale par laquelle un individu ou un groupe construit le réel et lui attribue une signification spécifique " (Abric 2014). Ainsi, au-delà des questions de dispositifs dits de participation, se pose cette interrogation : quelle place et quels rôles sont attribués aux représentations des acteurs et selon quelles conceptions ? Lintelligence $d u$ social supposerait la prise en compte de ces représentations, concept central dans le champ du social, en tant que processus de construction et d'échange de signification et corrélativement l'inscription des représentations des acteurs dans une perspective de participation à la Cité et aujourd'hui à des Cités, ce qui complexifie sérieusement la problématique identitaire. Si comme 
Bergson l'a posé, l'identité se construit et s'exprime dans le mouvement (Bergson 2014), une approche en termes d'intelligence $d u$ social ne réfute ni ne craint les mouvements de la pensée et de l'activité chez les acteurs; elle les considère comme des traductions et des appropriations qui sont autant de détournements de sens nécessaires, féconds et non nuisibles à l'organisation.

Puisque nous évoquons la place de l'acteur dans les organisations, rappelons l'analyse effectuée par Marcel Gauchet sur le glissement opéré ces trente ou quarante dernières années, en gros depuis le cours des années 70 , où l'individu a fait irruption dans les problématiques d'organisation. Ce dernier montre que cet individu a d'abord été envisagé sous l'angle d'une émancipation personnelle, une sorte de libération face au poids de l'institué, du structurel. Puis au cours des années 1980-90, ce serait plutôt une individualisation juridique qui se serait manifestée en termes de respect de droits, notamment au travail. Â propos des années 2000, Gauchet évoque un subjectivisme affectif et identitaire, une quête propre à une société expérientielle où la recherche d'émotions et de mise en scène de soi constituerait le moteur de l'agir social individuel (Gauchet 2004).

Ce qui nous paraît intéressant, tant dans l'approche d'Abric que celle de Gauchet - mais on pourrait citer bien d'autres contributions à ce sujet - c'est le caractère désormais résolument dynamique et processuel qui marque les recherches consacrées à l'individu et à son rapport aux organisations et à la société et ce sont bien ces dynamiques en cours que le concept d'intelligence du social tente d'approcher.

Afin de poursuivre cette tentative d'éclairage du concept, ajoutons qu'il questionne le rapport à l'évaluation dans les organisations : quelle signification est donnée à l'évaluation des activités et des acteurs ainsi qu'à la gratification et à la valorisation des expériences et des pratiques (lorsque cette valorisation est tout simplement envisagée) ?

C'est sans doute autour des enjeux liés à l'évaluation que nombre d'interprétations fonctionnalistes peuvent également apparaître lorsque la production formelle d'évaluation devient elle-même la finalité au détriment de la construction et du partage du sens des processus conduisant à l'action, comme nous avons pu le montrer, par exemple dans des dispositifs d'évaluation des activités en entreprise ou en administration, là où l'utilisation de l'outil de mesure dans des temps contraints constitue l'essentiel d'une évaluation limitée au contrôle à court terme et incapable de proposer des projections ou d'éclairer la prise de décisions. Lorsque remplir le tableau de bord devient la finalité de l'agir, la vision politique de l'action se trouve réduite ou annihilée et dans ce cas que fait-on socialement de l'évaluation : une question qui n'appellerait aucune réponse ? Au regard de quelles conceptions une autre acception de l'évaluation, notamment celle qui consisterait à attribuer de la valeur aux pensées et aux activités humaines se trouve-t-elle délaissée et quels 
sont les effets de ce délaissement sur les représentations des acteurs quant à leur possible inscription dans une organisation?

L'intelligence $d u$ social questionne encore le rapport à l'altérité. Acteur, auteur ou spectateur, disions-nous précédemment. Mais c'est aussi le problème du rapport à autrui en ce qui concerne sa motivation et sa capacité d'engagement ou encore de sa participation. J'évoquais tout à l'heure la faculté, la capabilité d'interprétation, de traduction accordée à autrui dans et par les organisations et la nécessité de cette traduction en vue d'une appropriation. Prenons le cas d'une organisation comme l'hôpital et considérons la situation du patient, posons comme hypothèse que la relation est un élément du soin de même que la participation du patient à l'ensemble du processus de soin. L'Autre est-il réputé présent et agissant dans cette relation ?

Considérons de même la notion de service dont on constate qu'elle est souvent reléguée à l'idée d'une fourniture pensée par son fournisseur alors que le problème est bien plus large et plus interactif puisqu'il n'y a service rendu que lorsque l'on peut observer une plus-value d'usage, de reconnaissance, de bien-être ou autre, chez le bénéficiaire dans le cadre d'une identité... identifiée. C'est une dimension problématique que nous avons pu relever lors d'une recherche menée dans le contexte des Allocations familiales à propos de la relation aux bénéficiaires : l'allocataire, c'est-à-dire le bénéficiaire selon le champ lexical de la Caisse d'Allocations familiales, ne se représente pas lui-même sous ce vocable, ce qui le conduit parfois à répondre lors d'une prise de contact « qu'il n'est pas locataire mais propriétaire »....

De même, nous nous penchons sur l'examen des pratiques observables de responsabilité, entendue ici comme perception pertinente des effets que chacun a sur autrui et sur l'organisation du fait de sa propre intervention. Qu'en est-il alors des discours sur la responsabilité : des discours incantatoires ou bien des discours co-construits en proximité des acteurs et des pratiques et pour quels effets ? Comment la responsabilité est-elle mise en récit dans les organisations et en quoi cette mise en récit témoigne-t-elle de la possibilité d'une intelligence $d u$ social comme principe agissant du vivre ensemble ?

De l'individu de droit que nous sommes tous, comme le soulignait Ulrich Beck, c'est-à-dire des individus appelés à chercher des solutions individuelles à des problèmes engendrés collectivement, par exemple la pollution (Beck, 2009), l'intelligence $d u$ social envisage la perspective et la facilitation de solutions co-construites, ce préfixe «co » impliquant des interactions permettant, comme l'a noté Castoriadis, de résoudre les problèmes engendrés socialement (Castoriadis 1999). De ce point de vue, les démarches dites de «projet " invitent à convoquer les acteurs concernés dès l'amont de ces derniers et non seulement en aval, selon le principe qui nous rappelle que "l'on comprend et l'on s'approprie mieux ce que l'on construit au moins en partie soi-même » (Bruner 2008). 
Penser l'intelligence $d u$ social, c'est encore questionner le rapport à la différence dans l'organisation. Comment les acteurs et leur organisation, à travers les choix politiques qu'elle pose, se représentent-ils les différences de parcours, de cultures, de logiques, de compétences ? Les appartenances croisées sont-elles favorisées ou considérées comme dangereuses pour la réussite des activités, voire la pérennité de l'organisation, cela induit-il des formes de gratification différenciées ? Le rapport à la différence est-il seulement évalué d'un point de vue prescriptif et structurel, lié par exemple à des statuts ou bien s'ouvre-t-il à d'autres facteurs ? Par exemple, assiste-ton à une hypersegmentation des formes de gratification comme dans cette administration centrale où la gestion de 17000 personnes intègre 950 régimes différents de primes?

En lien avec ces questions autour du rapport à la différence, figure le problème du rapport des acteurs et des organisations envers la gestion et la diffusion de l'information et donc envers la notion et la valeur de transparence, notamment en termes de possibilité d'accès à cette même information pour les différentes parties prenantes de l'organisation et de ses activités. Sans faire de la transparence un dogme au nom de je ne sais quelle performance ambitionnée par une sphère managériale parfois désireuse de convertir ses intentions en slogans, il s'agit d'examiner des canaux, des circuits, des supports ainsi que des politiques en matière d'information et de documentation. Ce sont donc des questions touchant aux conceptions de l'accessibilité, de la disponibilité et du sentiment d'équité envers l'information qui se posent ici et qui nous renvoient à un problème plus général, d'ailleurs souvent évoqué par des organisations en mal ou en quête de valorisation symbolique : celui des représentations individuelles et collectives de l'éthique au sein des organisations. L'éthique pourrait, à elle seule, constituer une entrée problématique pour l'ensemble de ce séminaire ; je me contenterai de faire un seul développement ou plutôt un lien à son sujet pour dire que l'étude des systèmes de valeur(s) dans les organisations constitue, en matière de recherche, un levier pour la compréhension de ce fameux sentiment d'appartenance, ici aussi tant réclamé par les managers, et qui, en fait, relève le plus souvent chez les acteurs de l'appropriation d'un but supra-ordonné, c'est-à-dire d'enjeux qui constituent des priorités considérées comme supérieures aux enjeux et centres d'intérêt individuels ou de groupes tels des groupes-métiers par exemple. En ce sens, l'appropriation, les transactions, l'accord qui se nouent autour de ces valeurs et de leur mise en œuvre, semble de nature à contribuer à forger une éthique de l'activité en contexte organisationnel et peut-être aussi la conception d'une organisation communicante.

Parvenu à ce point de ce modeste panorama des questions de recherche autour du concept d'intelligence $d u$ social, je dirai encore que celui-ci questionne la subsidiarité et les formes de solidarité dans les activités humaines. En me rapprochant du concept de coopération, tel que Sennett 
l'approche, il s'agit, par exemple, de comprendre les pratiques et une culture de veille sur l'Autre, telles qu'elles peuvent être observées dans certains secteurs professionnels comme la santé, l'éducation, l'artisanat mais aussi des activités de production de biens ou de services, au cœur de ces moments et de ces lieux où l'on essaie de se situer dans des interfaces de pratiques, de discours, de décision, là où le sens fait débat et est considéré comme négociable (Sennett 2014). Nous n'ignorons pas, cependant, la difficulté des acteurs à penser en termes d'interface et à s'y situer pour permettre des médiations sociales, cognitives ou autres ; c'est le cas, par exemple lorsque des transversalités sont appelées entre métiers et services dans des organisations où tout est construit et pensé de façon descendante.

Tout cela ne nous invite-t-il pas à poser le regard de la recherche, et notamment celui qui se fonde ici sur la rencontre entre les concepts de communication et d'organisation, sur les innovations, les tentatives des acteurs, sur ces savoirs d'action (Barbier 2011) construits par la pratique sociale car l'intelligence $d u$ social se façonne aussi dans les expériences du quotidien et dans leur expression en actes de langage et d'usages.

Afin d'esquisser quelques éléments de conclusion qui constitueront plutôt une mise en perspective, l'intelligence du social questionne la place et l'importance attribuées aux interstices, aux interfaces communicationnelles envers lesquels nous formons l'hypothèse selon laquelle elles seraient le creuset du sens collectif dans les organisations et donc les possibles de la communication organisée et organisante. De ce point de vue, les moments et les lieux de coconstruction, de concertation, de co-évaluation, de co-pilotage, ces moments et ces lieux qui renvoient à ce qui est dit, écrit, affiché, annoncé en termes de " co », ont-ils une place et une réalité dans des organisations généralement submergées par les exigences et les contraintes du quoi, du combien et du quand?

Nous aurons aperçu ici à quel point les problématiques soulevées par l'intelligence $d u$ social engagent une capacité à écouter les bruissements de l'organisation vivante, à en percevoir les signaux faibles (Ginzburg 2010) et non seulement les signaux forts, accessibles ou (re)connus. Et puis c'est aussi une invitation à donner la primauté aux questions touchant à la négociation du sens comme fondement et comme référent d'une possible prise de position ou de décision co-construite par et avec les parties prenantes de l'organisation, en quelque sorte l'hypothèse d'une paix possible au cœur des activités humaines...

À n'en pas douter, l'intelligence $d u$ social dans les organisations relève et soulève des problématiques d'ordre communicationnel... 


\section{BIBLIOGRAPHIE}

ABRIC J. C., (2014), Psychologie de la communication - Théories et méthodes, Armand Colin.

BARBIER J. M., (2011), Savoirs théoriques et savoirs d'action, Puf.

BAUMAN Z., (2007), Le présent liquide, Seuil et (2009), Identité, L'Herne.

BECK U., (2009), Pouvoir et contre-pouvoir à l'ère de la mondialisation, coll. Champs Essais, Flammarion.

BERGSON H., (2014), La pensée et le mouvant, Flammarion.

BERTHELOT J. M., (1997), L'intelligence du social, Puf.

BONNET J., BONNET R., RAICHVARG D., (2014), Communication et intelligence du social, 2 tomes, Coll. Communication et civilisation, L'Harmattan.

BRUNER J., (2008), L'éducation, entrée dans la culture, Retz.

CASTORIADIS B. (1999), L'institution imaginaire de la société, coll. Points essais, Seuil.

EHRENBERG A., (2010), La société du malaise - Le mental et le social, Odile Jacob

GAUCHET M., (2004), Un monde désenchanté ? L'Atelier et (2011), Le nouvel âge de l'individu in Philosophies et pensées de notre temps, éditions Sciences humaines, pp. 123-129.

GINZBURG C. coll. (2010), Mythes, emblèmes, traces - Morphologie et histoire, éditions Verdier.

LIPOVESTKY G. (2006), Les temps hypermodernes, Le livre de poche.

SEN A. (2010), L'idée de justice, éditions Flammarion.

SENNETT R. (2014), Ensemble - Pour une éthique de la coopération, Albin Michel.

Résumé : Traiter d'intelligence $d u$ social consiste à s'intéresser à ces postures, ces habiletés, ces modes et ces logiques d'intervention individuelles et collectives qui nourrissent, facilitent et rendent fécondes les interactions sociales dans les organisations. Mais l'héritage issu des modèles de la modernité industrielle rend-il possible cette prise en compte du social en faveur d'organisations communicantes ? Les notions et les pratiques de participation, de coopération, de disponibilité et d'accessibilité de l'information, de valorisation, d'altérité, de prise en compte de la différence, de négociation, et d'autres, sont approchées ici en tant que clés de lecture du vivre et agir ensemble au sein des organisations.

Mots-clés : coopération, intelligence du social, organisation communicante.

Abtract: The process of studying the intelligence of social relationships means a focus on these attitudes, relational skills, ways and logics of individual and collective intervention who provide social interactions in the organizations making them easier and more productive. But does the heritage from industrial modernity take into account the social relationships in 
favour of communicating organizations? In this article we deal with some notions et practices such as participation, cooperation, availability and accessibility of information or recognition, alterity, concern to different ways of being, negotiation and some others who are taken as keys to understanding of a way of living and acting together within the context of organizations.

Keywords: cooperation, intelligence of social relationships, communicating organization. 